\title{
Tandem High-dose Chemotherapy and Autologous Stem Cell Transplantation in Children with Brain Tumors : Review of Single Center Experience
}

\author{
Ki Woong Sung, M.D., Ph.D., ${ }^{1}$ Do Hoon Lim, M.D., Ph.D., ${ }^{2}$ Hyung Jin Shin, M.D., Ph.D. ${ }^{3}$ \\ Departments of Pediatrics, ${ }^{1}$ Radiation Oncology, ${ }^{2}$ Neurosurgery, ${ }^{3}$ Samsung Medical Center, Sungkyunkwan University School of Medicine, \\ Seoul, Korea
}

The prognosis of brain tumors in children has improved for last a few decades. However, the prognosis remains dismal in patients with recurrent brain tumors. The outcome for infants and young children in whom the use of radiotherapy (RT) is very limited because of unacceptable long-term adverse effect of RT remains poor. The prognosis is also not satisfactory when a large residual tumor remains after surgery or when leptomeningeal seeding is present at diagnosis. In this context, a strategy using high-dose chemotherapy and autologous stem cell transplantation (HDCT/auto-SCT) has been explored to improve the prognosis of recurrent or high-risk brain tumors. This strategy is based on the hypothesis that chemotherapy dose escalation might result in improvement in survival rates. Recently, the efficacy of tandem HDCT/auto-SCT has been evaluated in further improving the outcome. This strategy is based on the hypothesis that further dose escalation might result in further improvement in survival rates. At present, the number of studies employing tandem HDCT/auto-SCT for brain tumors is limited. However, results of these pilot studies suggest that tandem HDCT/auto-SCT may further improve the outcome. In this review, we will summarize our single center experience with tandem HDCT/auto-SCT for recurrent or high-risk brain tumors.

Key Words : Brain neoplasms · Child · High-dose chemotherapy · Autologous stem cell transplantation.

\section{INTRODUCTION}

The prognosis of brain tumors in children has improved for the last 2-3 decades. However, the prognosis remains dismal in patients with relapsed brain tumors ${ }^{3,18}$. The outcome for infants and young children in whom the use of radiotherapy (RT) is very limited because of unacceptable long-term adverse effect of RT remains poor ${ }^{5,6,20)}$. The prognosis is also not satisfactory when a large residual tumor remains after surgery or when leptomeningeal seeding is present at diagnosis ${ }^{13,29,30)}$. The prognosis of high-grade gliomas (HGGs) remains very poor ${ }^{8,923)}$. In addition, late adverse effects from RT, particularly craniospinal RT, is unavoidable ${ }^{5,20)}$.

A strategy using high-dose chemotherapy and autologous stem cell transplantation (HDCT/auto-SCT) has been explored to improve the prognosis of patients with high-risk solid tumors $^{2,17)}$. This strategy is based on the hypothesis that dose escalation might improve the survival of children. The results of

- Received : February 13, 2018 •Revised : March 2, 2018 •Accepted : March 8, 2018

- Address for reprints : Ki Woong Sung, M.D., Ph.D.

Department of Pediatrics, Samsung Medical Center, Sungkyunkwan University School of Medicine, 81 Irwon-ro, Gangnam-gu, Seoul 06351, Korea Tel : +82-2-3410-3529, Fax : +82-2-3410-0043, E-mail : kwsped@skku.edu

This is an Open Access article distributed under the terms of the Creative Commons Attribution Non-Commercial License (http://creativecommons.org/licenses/by-nc/4.0) which permits unrestricted non-commercial use, distribution, and reproduction in any medium, provided the original work is properly cited. 
randomized trials comparing HDCT/auto-SCT with chemotherapy alone in patients with high-risk neuroblastoma showed a better event-free survival (EFS) in the HDCT/auto-SCT arm than in the continuous chemotherapy arm tumors ${ }^{2,17)}$. Thus, HDCT/auto-SCT has been the standard consolidation treatment for high-risk neuroblastoma. Following the success in high-risk neuroblastoma, the efficacy of HDCT/auto-SCT has been explored to improve the prognosis of relapsed or highrisk brain tumors and/or to avoid or minimize $\mathrm{RT}^{4,7,10,16,19,22)}$.

Although the prognosis of relapsed or high-risk solid tumors has improved by using HDCT/auto-SCT, it remains still unsatisfactory. The main cause of failure after single HDCT/autoSCT is relapse or tumor progression rather than treatment-related mortality (TRM). Thus, some investigators have explored the efficacy of tandem HDCT/auto-SCT to further improve the outcome ${ }^{11,12,24)}$. This strategy is based on the hypothesis that further dose escalation might result in further improvement in survival rates. Results from a small number of pilot studies suggest that further dose escalation might result in further improvements in the EFS. Our center has used tandem HDCT/ auto-SCT since 2005 for the treatment of relapsed or high-risk brain tumors in children and adolescents. Here, we briefly review our experience with tandem HDCT/auto-SCT for brain tumors (Table 1).

\section{HDCT/AUTO-SCT FOR RELAPSED BRAIN TUMORS IN CHILDREN AND ADOLESCENTS}

\section{Relapsed medulloblastoma : KSPNO S-053 study ${ }^{21)}$}

The outcome of relapsed medulloblastoma (MB) remains dismal with conventional treatment modalities. Thus, the Korean Society of Pediatric Neuro-Oncology (KSPNO) investigated the efficacy and toxicity of HDCT/auto-SCT for improving the outcomes of patients with relapsed MB. A total of 15 patients with relapsed MB were enrolled in the KSPNO S-053 study from 2005 to 2007. All patients received approximately four cycles of salvage chemotherapy after relapse. Thirteen underwent HDCT/auto-SCT; carboplatin+thiotepa+etoposide (CTE) and cyclophosphamide+melphalan (CyM) regimens were used for the first HDCT and second HDCT, respectively, and seven underwent second HDCT. One TRM due to hepatic veno-occlusive disease (VOD) occurred during first HDCT but second HDCT was tolerable with no further TRM. The 3-year overall survival (OS) and event-free survival (EFS) rates ( $\pm 95 \%$ confidence intervals) were $33.3 \pm 12.2 \%$ and $26.7 \pm 11.4 \%$, respectively. When the analysis was confined to only patients who achieved a complete response (CR) or partial response (PR) prior to HDCT, the probability of 3-year OS rates was $40.0 \pm 15.5 \%$. No patients with stable disease (SD) or progressive disease $(\mathrm{PD})$ survived. This study showed that the tumor status before HDCT/auto-SCT had a crucial impact on the outcome after HDCT/auto-SCT. These results will provide additional evidence for a rational selection of patients for HDCT/auto-SCT after relapse/progression, considering significant risk and cost accompanying HDCT/auto-SCT.

\section{Relapsed germ cell tumors : KSPNO S-053 study ${ }^{1)}$}

The outcomes in patients with relapsed or progressed central nervous system (CNS) germ cell tumors (CNS-GCTs), especially those with non-germinomatous GCTs (NGGCTs), remain poor, and there is no clear consensus on management of these tumors. In this context, the KSPNO conducted a prospective, multicenter clinical study to evaluate the feasibility and effectiveness of tandem HDCT/auto-SCT in treating children with relapsed or progressed CNS-GCTs. Eleven patients with NGGCTs and nine patients with germinomas were enrolled. Patients received between two and eight cycles of conventional chemotherapy prior to HDCT/auto-SCT with or without RT. Overall, 16 patients proceeded to the first HDCT/auto-SCT, and nine proceeded to the second HDCT/auto-SCT. CTE and CyM regimens were used for the first and second HDCT, respectively. Toxicities during HDCT/auto-SCT were acceptable, and there was no TRM. Twelve patients experienced relapse or progression again; however, four patients with germinomas remain alive after subsequent RT. Therefore, a total of 12 patients (four NGGCTs and eight germinomas) remain alive with a median follow-up of 47 months after initial relapse or progression. The probability of 3 -year OS was $59.1 \pm 11.2 \%$ (36.4 $\pm 14.5 \%$ for NGGCTs vs. $88.9 \pm 10.5 \%$ for germinomas, $P=0.028$ ). RT, particularly craniospinal RT, was associated with a better tumor response prior to HDCT/auto-SCT and a better final outcome. This study showed that tandem HDCT/auto-SCT is a feasible approach in the treatment of relapsed or progressed CNS-GCTs. Further studies with larger cohorts of patients are needed to elucidate the possible role of HDCT/auto-SCT in the treatment of relapsed or progressed CNS-GCTs. 
Tandem HDCT/Auto-SCT for Brain Tumors | Sung KW, et al.

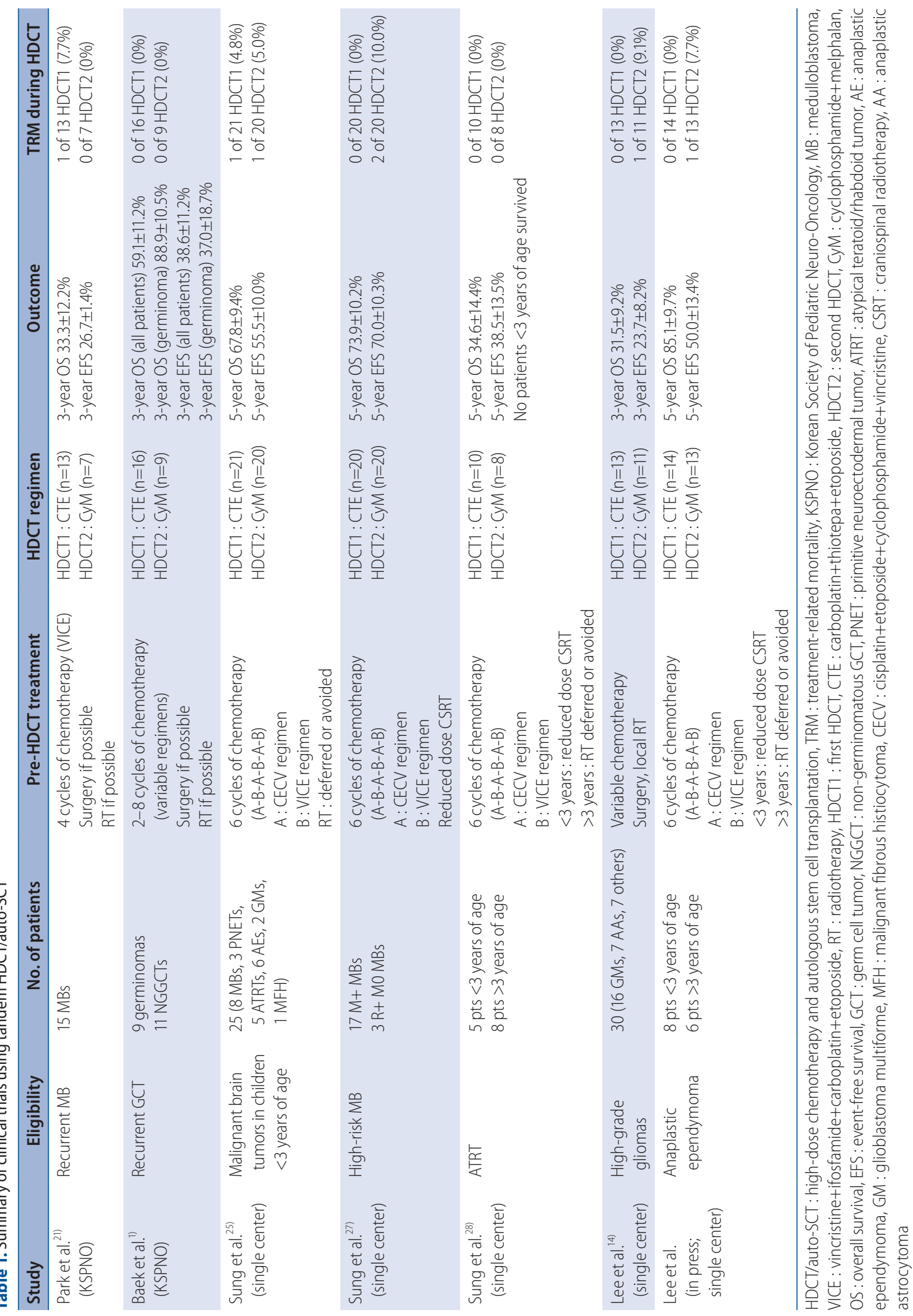




\section{HDCT/auto-SCT for brain tumors in very young children : single center experience ${ }^{25)}$}

Treatment of malignant brain tumors in young children is challenging because RT is used minimally due to the risk of functional impairment of the developing brain, and late adverse effects, including global reduction in the intelligence quotient, cognitive deficits, and neuroendocrine dysfunction. In an effort to minimize these effects, a number of institutions and national groups have adopted chemotherapy-based strategies designed to avoid or delay RT, though outcomes have been largely unsatisfactory. Clinical trials using HDCT/auto-SCT for treatment of infants and young children with malignant brain tumors have shown that it is possible to avoid or defer RT until 3 years of age while maintaining or improving survival rates.

For this reason, we prospectively evaluated the feasibility and effectiveness of tandem HDCT/auto-SCT in children under 3 years of age with malignant brain tumors. This treatment strategy aimed to simultaneously improve survival and reduce the risk of significant late adverse effects of RT. A total of 25 children under 3 years of age received tandem HDCT/auto-SCT following six cycles of induction chemotherapy. RT was either not given or deferred until 3 years of age if the patient achieved CR after tandem HDCT/auto-SCT. Local RT was given only if gross residual tumor $\left(>1.5 \mathrm{~cm}^{2}\right)$ remained after surgery, and craniospinal RT was given only if leptomeningeal seeding was present at the time of diagnosis. Tumors relapsed or progressed in nine patients (five during induction treatment), and two of these patients survived after receiving salvage treatment, including RT. Two patients died due to toxicities during tandem HDCT/auto-SCT. A total of 16 patients survived to a median follow-up period of 52 months from the time of diagnosis. Four of these patients did not receive RT, two received local RT alone, three received craniospinal $\mathrm{RT}$ alone and seven received both. The 5-year OS and EFS rates were $67.8 \pm 9.4 \%$ and $55.5 \pm 10.0 \%$, respectively. Neuroendocrine and neurocognitive functions evaluated at 3 years after tandem HDCT/auto-SCT were acceptable. The results of this study indicate that tandem HDCT/auto-SCT may improve survival in young children with malignant brain tumors with an acceptable level of risk of long-term toxicity.

\section{HDCT/AUTO-SCT FOR HIGH-RISK EMBRYONAL TUMORS}

\section{High-risk MB : single center experience ${ }^{27)}$}

$\mathrm{MB}$ is the most common malignant brain tumor in children, accounting for $20 \%$ of childhood brain tumors. The standard treatment of MB consists of surgery; RT, including craniospinal RT; and chemotherapy. The conventional doses of RT have been around 36 Gy to the craniospinal axis combined with a boost of 18-20 Gy to the posterior fossa, for a total dose of 5456 Gy. Children who survive MB are at risk for various late adverse effects primarily attributable to RT, particularly craniospinal RT. The prognosis for high-risk MB (defined by metastasis and/or postoperative residual tumor $>1.5 \mathrm{~cm}^{2}$ ) is unsatisfactory, despite standard-dose craniospinal RT; 5-year survival is $<55 \%$.

HDCT/auto-SCT has been used to treat high-risk or recurrent MBs. Some investigators have applied this treatment modality in relapsed/progressed cases, and some reports exist that use this strategy as a first-line treatment for newly diagnosed MBs. Gajjar and colleagues have reported the results of a prospective study (St. Jude MB 96 study) using tandem HDCT/ auto-SCT in patients with high-risk MB. In their study, patients received 36.0-39.6 Gy of craniospinal RT followed by four cycles of HDCT/auto-SCT, and the 5-year EFS rate was 70\%. The EFS rate was encouraging; however, late adverse effects from dose-intense chemotherapy might potentiate late adverse effects from conventional RT.

For this reason, we assessed the feasibility and effectiveness of reduced-dose craniospinal RT followed by tandem HDCT/ auto-SCT in reducing late adverse effects without jeopardizing survival among children with high-risk MB. From 2005 through 2010, twenty consecutive children aged $>3$ years with high-risk $\mathrm{MB}$ were assigned to receive two cycles of pre-RT chemotherapy, reduced craniospinal RT (23.4 or 30.6 Gy) combined with local RT to the primary site (total $54.0 \mathrm{~Gy}$ ), and four cycles of post-RT chemotherapy followed by tandem HDCT/auto-SCT. CTE and CyM regimens were used for the first and second HDCT, respectively. Of 20 patients with high-risk MB, 17 had metastatic disease and three had a postoperative residual tumor $>1.5 \mathrm{~cm}^{2}$ without metastasis. The tumor relapsed/progressed in four patients, and two patients died from toxicities during the second HDCT/auto-SCT. Therefore, 14 patients remained event-free at a median follow-up of 46 months from di- 
agnosis. The probability of 5-year EFS was $70.0 \pm 10.3 \%$ for all patients and $70.6 \pm 11.1 \%$ for patients with metastases. Late adverse effects evaluated at a median of 36 months after tandem HDCT/auto-SCT were acceptable. This study suggests that, in children with high-risk MB, the dose of craniospinal RT might be reduced without jeopardizing survival by using tandem HDCT/auto-SCT.

\section{Atypical teratoid/rhabdoid tumors : single center experience $^{28)}$}

CNS atypical teratoid/rhabdoid tumors (ATRTs) are highly malignant and are associated with significantly worse OS than other embryonal tumors. The very poor prognosis of ATRT in young children may be related to the limited use of RT because of the risks of functional impairment of the developing brain and late adverse effects. Multiple chemotherapeutic approaches have been attempted to defer RT; however, OS remains dismal. For older children, a few studies have suggested that outcome might be improved with standard-dose craniospinal RT and high-dose alkylator-based chemotherapy. However, various late adverse effects primarily attributable to RT, particularly craniospinal RT, were unavoidable.

For this reason, we prospectively evaluated the effectiveness of tandem HDCT/auto-SCT in improving the survival of patients with ATRT while reducing the risks of late adverse effects from RT. For young children ( $<3$ years old), tandem HDCT/ auto-SCT was administered after six cycles of induction chemotherapy. RT was deferred until after 3 years of age unless the tumor showed relapse or progression. For older patients $(>3$ years old), RT including reduced dose craniospinal RT (23.4 or $30.6 \mathrm{~Gy}$ ) was administered either after two cycles of induction chemotherapy or after surgery, and tandem HDCT/auto-SCT was administered after six cycles of induction chemotherapy. A total of 13 patients (five young and eight older) were enrolled from 2004 to 2012. Eight patients, including all five young patients, had metastatic disease at diagnosis. Six patients (four young and two older) experienced progression before initiation of RT, and seven were able to proceed to HDCT/auto-SCT without progression during induction treatment. Three of six patients who experienced progression during induction treatment underwent HDCT/auto-SCT as salvage treatment. All five young patients died from disease progression. However, four of the eight older patients remain progression-free with a median follow-up period of 64 months. Treatment-related late toxicities were acceptable. This study showed that the required dose of craniospinal RT might be reduced in older patients if the intensity of chemotherapy is increased. However, early administration of RT should be considered to prevent early progression in young patients.

\section{HDCT/AUTO-SCT FOR OTHER HIGH-RISK BRAIN TUMORS}

\section{High-grade gliomas : single center experience ${ }^{14)}$}

While HGGs represent one of the most common CNS tumors in adults, HGGs are less common in children and adolescents. HGGs include a variety of heterogeneous lesions with differing histologies, but the most common histologies are anaplastic astrocytoma (World Health Organization [WHO] grade III) and glioblastoma (WHO grade IV). The prognosis of HGGs has been very poor and there is no universally accepted standard care for HGGs in children. Previous children's cancer group study showed the effectiveness of adjuvant chemotherapy but current conventional therapies are not yet sufficient for survival. In adults, concomitant temozolomide and RT prolonged survival duration and is now considered the standard of treatment; however, multiple studies failed to demonstrate the benefits of TMZ on long-term survival in children. In addition, prolongation of survival, not cure, is less meaningful in children than in adults.

For this reason, we reported our experience with HDCT/auto-SCT for HGGs in 30 children and adolescents (16 glioblastomas, seven anaplastic astrocytomas, and seven other HGGs) between 2006 and 2015. Gross or near total resection was possible in 11 patients. Front-line treatment after surgery was RT in 14 patients and chemotherapy in the remaining 16 patients including three patients less than 3 years of age. Eight of 12 patients who remained progression free and five of the remaining 18 patients who experienced progression during induction treatment underwent the first HDCT/auto-SCT with CTE regimen and 11 of them proceeded to the second HDCT/auto-SCT with $\mathrm{CyM}$ regimen. One patient died from hepatic VOD during the second HDCT/auto-SCT; otherwise, toxicities were manageable. Four patients in CR and three of seven patients in PR or second PR at the first HDCT/auto-SCT remained event free : however, two patients with progressive tumor experienced progression again. The probability of 3-year OS after the first HDCT/ 
auto-SCT in 11 patients in CR, PR, or second PR was $58.2 \pm 16.9 \%$. Tumor status at the first HDCT/auto-SCT was the only significant factor for outcome after HDCT/auto-SCT. This study suggests that the outcome of HGGs in children and adolescents after HDCT/auto-SCT is encouraging if the patient could achieve CR or PR before HDCT/auto-SCT.

\section{Anaplastic ependymoma : single center experience (in press)}

Ependymomas occur more frequently in children than in adults. Ependymomas have been classified as WHO grades I, II, and III based on the histologic findings and surgical resection with or without adjuvant RT has been the mainstay therapy. The effectiveness of chemotherapy is controversial; however, some studies have suggested that chemotherapy may be beneficial for ependymomas that are not completely resected, making it possible to either delay RT without compromising survival or facilitate resection of residual tumors. The potential beneficial effects of chemotherapy are expected to be more prominent in high-grade ependymomas with anaplastic features than in low-grade ependymomas. The results of our early study involving five very young children with anaplastic ependymomas have also suggested that tandem HDCT/auto-SCT is a feasible therapeutic option that exhibits acceptable toxicities and may improve the survival of patients ${ }^{26)}$.

Recently, we have evaluated the results of multimodal treatment that includes tandem HDCT/auto-SCT, not only in this young population, but also in older children with anaplastic ependymomas, and with longer durations of follow-up. Fourteen patients with anaplastic ependymomas diagnosed from 2006 to 2014 were reviewed. Six cycles of induction chemotherapy were administered to all patients before they underwent tandem HDCT/auto-SCT. Patients who were older than 3 years of age received RT after two cycles of induction chemotherapy. In patients under 3 years of age, RT was either omitted or delayed until they reached 3 years of age, if the patients experienced CR after tandem HDCT/auto-SCT. All patients, including two who experienced disease progression during induction treatment, underwent the first HDCT/auto-SCT, and 13 subsequently underwent the second HDCT/auto-SCT. One patient died from hepatic VOD during the second HDCT/auto-SCT; other toxicities occurring during tandem HDCT/auto-SCT were manageable. Relapses or progression occurred in seven patients, and five of them remain alive after salvage treatment, including sur- gery and RT. The 5-year OS and EFS rates were $85.1 \pm 9.7 \%$ and $50.0 \pm 13.4 \%$, respectively. The results of this study suggest that multimodal treatment including tandem HDCT/auto-SCT could be a feasible option for improving survival in children with anaplastic ependymomas.

\section{Toxicities of tandem HDCT/auto-SCT : single center experience $^{15)}$}

Recently, the number of studies examining the efficacy of tandem HDCT/auto-SCT to further improve the outcome of high-risk or recurrent brain tumors is increasing. However, tandem HDCT/auto-SCT might be associated with greater toxicity and higher TRM rates than single HDCT/auto-SCT. However, studies addressing the toxicity associated with tandem HDCT/auto-SCT for brain tumors, particularly during the second HDCT/auto-SCT, are limited.

For this reason, we evaluated the toxicity of tandem HDCT/ auto-SCT using the CTE and CyM regimens that we have used regularly since 2005 . We also evaluated risk factors for morbidity and mortality during tandem HDCT/auto-SCT, particularly during the second HDCT/auto-SCT. A total of 109 patients who underwent the first HDCT/auto-SCT and 100 who proceeded to the second HDCT/auto-SCT between 2005 and 2013 were included. Hematologic recovery was rapid during both the first and second HDCT/auto-SCT. In the first HDCT/auto-SCT, mucositis-related gastrointestinal toxicity was frequent, and two $(1.8 \%)$ patients died from toxicity (one from hepatic VOD and the other from sepsis). In the second HDCT/auto-SCT, mucositis-related toxicity was milder than in the first round. However, hepatic VOD frequency was high (20.0\%), and six (6.0\%) patients died from toxicity (four from hepatic VODs, one from asphyxia, and one from sepsis). Multivariate analysis indicated that age younger than 8 years was the only significant predictor for hepatic VOD. All six patients who died from toxicity during the second HDCT/auto-SCT were younger than 9 years of age. This study demonstrates that tandem HDCT/auto-SCT using $\mathrm{CTE} / \mathrm{CyM}$ regimens was generally feasible. However, dose reduction during the second HDCT/auto-SCT in young children might be needed to decrease the death rate from toxicity.

\section{DISCUSSION}

Our experience with tandem HDCT/auto-SCT as salvage 
treatment for relapsed brain tumors or as front-line treatment for high-risk tumors suggests that tandem HDCT/auto-SCT might improve survival outcomes or reduce RT dose without jeopardizing survival rates. However, there are a few significant limitations or clinical problems to be solved.

First, risk stratifications for inclusion criteria in our previous studies were based on the conventional clinical parameters (age, the presence of metastasis, significant postoperative residual tumor, or conventional histology). Recent efforts at stratifying brain tumors on the basis of their molecular features have subdivided brain tumors into various distinct molecular subgroups characterized by disparate transcriptional signatures, mutational spectra, copy number profiles, and clinical features. Molecular stratification of brain tumors with use of a gene expression-based approach will improve current risk stratification. In the next phase of clinical trial employing HDCT/auto-SCT, the selection of patients will be based also on these data, thus potentially avoiding intensive treatment regimen to some of those patients previously considered as high-risk. Second, the number of patients enrolled in our single arm pilot studies was low due to the rarity of each brain tumor. In addition, there is no study comparing the effectiveness and toxicity between single and tandem HDCT/auto-SCT in patients with brain tumors. Therefore, prospective randomized controlled studies with a larger cohort of patients are needed to confirm the results of our single arm pilot studies. Nation-wide trials might be necessary. Third, tandem HDCT/auto-SCT might be associated with greater toxicity and higher TRM rates than single HDCT/autoSCT. Therefore, long-term follow-up is needed to evaluate whether the survival benefits from tandem HDCT/auto-SCT or benefits from RT dose reduction outweigh the long-term risks of tandem HDCT/auto-SCT. Fourth, we have used only CTE and CyM regimens for tandem HDCT/auto-SCT in order to overcome possible drug resistance and avoid overlapping toxicities. However, toxicities during the second HDCT/auto-SCT were significant and some patients died from toxicities, particularly hepatic VOD. Therefore, dose modification of current regimen or employment of other HDCT regimen might be considered to reduce toxicities during the second HDCT/auto-SCT. Fifth, our studies for relapsed brain tumors showed that the tumor status before HDCT/auto-SCT had a crucial impact on the survival after HDCT/auto-SCT. The significance of local control via surgery or RT has been previously suggested in several types of solid tumors. In our experience with relapsed brain tumors, debulking surgery and RT after relapse/progression were also prognostic factors, further highlighting the importance of local control. Thus, surgery (repeated if necessary) and RT must be performed whenever feasible in order to reduce the tumor burden as much as possible prior to HDCT/auto-SCT. In addition, new chemotherapeutic drug such as target agents or immune regulatory agents might be needed to improve the response before HDCT/auto-SCT.

\section{CONCLUSION}

In conclusion, the results of our single center pilot studies suggest that tandem HDCT/auto-SCT might improve survival outcomes or reduce RT dose without jeopardizing survival rates in patients with relapsed or high-risk brain tumor. Prospective randomized controlled studies with a larger cohort of patients are needed to confirm the results of our studies.

\section{CONFLICTS OF INTEREST}

No potential conflict of interest relevant to this article was reported.

\section{INFORMED CONSENT}

This type of study does not require informed consent.

\section{References}

1. Baek HJ, Park HJ, Sung KW, Lee SH, Han JW, Koh KN, et al. : Myeloablative chemotherapy and autologous stem cell transplantation in patients with relapsed or progressed central nervous system germ cell tumors: results of Korean Society of Pediatric Neuro-Oncology (KSPNO) S-053 study. J Neurooncol 114 : 329-338, 2013

2. Berthold F, Boos J, Burdach S, Erttmann R, Henze G, Hermann J, et al. : Myeloablative megatherapy with autologous stem-cell rescue versus oral maintenance chemotherapy as consolidation treatment in patients with high-risk neuroblastoma: a randomised controlled trial. Lancet Oncol $6: 649-658,2005$

3. Bouffet E, Doz F, Demaille MC, Tron P, Roche H, Plantaz D, et al. : Improving survival in recurrent medulloblastoma: earlier detection, better treatment or still an impasse? Br J Cancer 77 : 1321-1326, 1998 
4. Chi SN, Gardner SL, Levy AS, Knopp EA, Miller DC, Wisoff JH, et al. : Feasibility and response to induction chemotherapy intensified with highdose methotrexate for young children with newly diagnosed high-risk disseminated medulloblastoma. J Clin Oncol 22 : 4881-4887, 2004

5. Copeland DR, deMoor C, Moore BD 3rd, Ater JL : Neurocognitive development of children after a cerebellar tumor in infancy: a longitudinal study. J Clin Oncol 17 : 3476-3486, 1999

6. Duffner PK, Horowitz ME, Krischer JP, Friedman HS, Burger PC, Cohen ME, et al. : Postoperative chemotherapy and delayed radiation in children less than three years of age with malignant brain tumors. $\mathbf{N}$ Engl J Med 328 : 1725-1731, 1993

7. Fangusaro J, Finlay J, Sposto R, Ji L, Saly M, Zacharoulis S, et al. : Intensive chemotherapy followed by consolidative myeloablative chemotherapy with autologous hematopoietic cell rescue (AuHCR) in young children with newly diagnosed supratentorial primitive neuroectodermal tumors (sPNETS): report of the Head Start I and II experience. Pediatr Blood Cancer $50: 312-318,2008$

8. Finlay JL, Boyett JM, Yates AJ, Wisoff JH, Milstein JM, Geyer JR, et al. : Randomized phase III trial in childhood high-grade astrocytoma comparing vincristine, lomustine, and prednisone with the eight-drugs-in-1-day regimen. Childrens cancer group. J Clin Oncol 13 : 112-123, 1995

9. Finlay $\mathrm{JL}$, Zacharoulis $S$ : The treatment of high grade gliomas and diffuse intrinsic pontine tumors of childhood and adolescence: a historical - and futuristic - perspective. J Neurooncol 75 : 253-266, 2005

10. Gardner SL, Asgharzadeh S, Green A, Horn B, McCowage G, Finlay J : Intensive induction chemotherapy followed by high dose chemotherapy with autologous hematopoietic progenitor cell rescue in young children newly diagnosed with central nervous system atypical teratoid rhabdoid tumors. Pediatr Blood Cancer 51 : 235-240, 2008

11. George RE, Li S, Medeiros-Nancarrow C, Neuberg D, Marcus K, Shamberger RC, et al. : High-risk neuroblastoma treated with tandem autologous peripheral-blood stem cell-supported transplantation: long-term survival update. J Clin Oncol 24 : 2891-2896, 2006

12. Kletzel M, Katzenstein HM, Haut PR, Yu AL, Morgan E, Reynolds M, et al. : Treatment of high-risk neuroblastoma with triple-tandem high-dose therapy and stem-cell rescue: results of the Chicago Pilot II Study. J Clin Oncol 20 : 2284-2292, 2002

13. Kortmann RD, Kühl J, Timmermann B, Mittler U, Urban C, Budach V, et al. : Postoperative neoadjuvant chemotherapy before radiotherapy as compared to immediate radiotherapy followed by maintenance chemotherapy in the treatment of medulloblastoma in childhood: results of the German prospective randomized trial HIT '91. Int J Radiat Oncol Biol Phys 46 : 269-279, 2000

14. Lee JW, Lim DH, Sung KW, Lee HJ, Yi ES, Yoo KH, et al. : Tandem highdose chemotherapy and autologous stem cell transplantation for highgrade gliomas in children and adolescents. J Korean Med Sci 32 : 195-203, 2017

15. Lee SH, Son MH, Sung KW, Choi YB, Lee NH, Yoo KH, et al. : Toxicity of tandem high-dose chemotherapy and autologous stem cell transplantation using carboplatin-thiotepa-etoposide and cyclophosphamidemelphalan regimens for malignant brain tumors. J Neurooncol 120 :
507-513, 2014

16. Mason WP, Grovas A, Halpern S, Dunkel IJ, Garvin J, Heller G, et al. : Intensive chemotherapy and bone marrow rescue for young children with newly diagnosed malignant brain tumors. J Clin Oncol 16 : 210-221, 1998

17. Matthay KK, Reynolds CP, Seeger RC, Shimada H, Adkins ES, Haas-Kogan $D$, et al. : Long-term results for children with high-risk neuroblastoma treated on a randomized trial of myeloablative therapy followed by 13-cis-retinoic acid: a children's oncology group study. J Clin Oncol 27 : 1007-1013, 2009

18. Merchant TE, Sherwood SH, Mulhern RK, Rose SR, Thompson SJ, Sanford RA, et al. : CNS germinoma: disease control and long-term functional outcome for 12 children treated with craniospinal irradiation. Int J Radiat Oncol Biol Phys 46 : 1171-1176, 2000

19. Modak S, Gardner S, Dunkel IJ, Balmaceda C, Rosenblum MK, Miller DC, et al. : Thiotepa-based high-dose chemotherapy with autologous stemcell rescue in patients with recurrent or progressive CNS germ cell tumors. J Clin Oncol 22 : 1934-1943, 2004

20. Mulhern RK, Merchant TE, Gajjar A, Reddick WE, Kun LE : Late neurocognitive sequelae in survivors of brain tumours in childhood. Lancet Oncol 5 : 399-408, 2004

21. Park JE, Kang J, Yoo KH, Sung KW, Koo HH, Lim DH, et al. : Efficacy of high-dose chemotherapy and autologous stem cell transplantation in patients with relapsed medulloblastoma: a report on the Korean Society for Pediatric Neuro-Oncology (KSPNO)-S-053 study. J Korean Med Sci 25 : 1160-1166, 2010

22. Pérez-Martínez A, Lassaletta A, González-Vicent M, Sevilla J, Díaz MA, Madero $L$ : High-dose chemotherapy with autologous stem cell rescue for children with high risk and recurrent medulloblastoma and supratentorial primitive neuroectodermal tumors. J Neurooncol 71 : 33-38, 2005

23. Sposto R, Ertel IJ, Jenkin RD, Boesel CP, Venes JL, Ortega JA, et al. : The effectiveness of chemotherapy for treatment of high grade astrocytoma in children: results of a randomized trial. A report from the Childrens Cancer Study Group. J Neurooncol 7 : 165-177, 1989

24. Sung KW, Lee SH, Yoo KH, Jung HL, Cho EJ, Koo HH, et al. : Tandem highdose chemotherapy and autologous stem cell rescue in patients over 1 year of age with stage 4 neuroblastoma. Bone Marrow Transplant 40 : 37-45, 2007

25. Sung KW, Lim DH, Lee SH, Yoo KH, Koo HH, Kim JH, et al. : Tandem highdose chemotherapy and auto-SCT for malignant brain tumors in children under 3 years of age. Bone Marrow Transplant 48 : 932-938, 2013

26. Sung KW, Lim DH, Lee SH, Yoo KH, Koo HH, Kim JH, et al. : Tandem high-dose chemotherapy and autologous stem cell transplantation for anaplastic ependymoma in children younger than 3 years of age. J Neurooncol $107: 335-342,2012$

27. Sung KW, Lim DH, Son MH, Lee SH, Yoo KH, Koo HH, et al. : Reduceddose craniospinal radiotherapy followed by tandem high-dose chemotherapy and autologous stem cell transplantation in patients with highrisk medulloblastoma. Neuro Oncol 15 : 352-359, 2013

28. Sung KW, Lim DH, Yi ES, Choi YB, Lee JW, Yoo KH, et al. : Tandem high- 
Tandem HDCT/Auto-SCT for Brain Tumors | Sung KW, et al.

dose chemotherapy and autologous stem cell transplantation for atypical teratoid/rhabdoid tumor. Cancer Res Treat 48 : 1408-1419, 2016

29. Taylor RE, Bailey CC, Robinson KJ, Weston CL, Walker DA, Ellison D, et al. : Outcome for patients with metastatic (M2-3) medulloblastoma treated with SIOP/UKCCSG PNET-3 chemotherapy. Eur J Cancer 41 : 727-734, 2005
30. Zeltzer PM, Boyett JM, Finlay JL, Albright AL, Rorke LB, Milstein JM, et al. : Metastasis stage, adjuvant treatment, and residual tumor are prognostic factors for medulloblastoma in children: conclusions from the Children's Cancer Group 921 randomized phase III study. J Clin Oncol 17 : 832845, 1999 